\title{
Trasplante haploidéntico con ciclofosfamida postrasplante (TMO-haplo-ciclo-pos) en leucemia linfoide aguda (LLA). Resultados preliminares de una serie de 22 pacientes, una de las más grandes reportadas
}

- Amado Karduss-Urueya, Rosendo Pérez, Gloria Suárez, José Betancur, Mónica Ramírez, Alejo Jiménez,

Pedro Reyes, Luis Rodolfo Gómez-Wollf, Giovanni Ruiz, Angélica Cardona

Programa de Trasplante de Médula Ósea, Instituto de Cancerología, Clínica Las Américas,

Unidad de Cuidados Intensivos Pediátricos, Clínica Las Américas,

Unidad de Investigación Instituto de Cancerología, Clínica Las Américas, Medellín (Antioquia).

Contacto: inscance@une.net.co

Introducción y objetivos. Las series publicadas acerca del uso de TMO-haplo-ciclo-pos tienen escasa representación de casos de LLA y ausencia de reporte de los desenlaces específicos en esta enfermedad. Presentamos nuestra experiencia.

Materiales y métodos. La fuente celular fue sangre periférica, el acondicionamiento: fludarabina $150 \mathrm{mg} / \mathrm{m}^{2}$, busulfán $6-8 \mathrm{mg} / \mathrm{kg}$ y TBI 400 cGy (flu-bu-TBI), o fludarabina $150 \mathrm{mg} / \mathrm{m}^{2}$, melfalán 100-140 $\mathrm{mg} / \mathrm{m}^{2}$ y TBI 200-400 cGy (flu-mel-TBI); en los días +3 y +4 se administró ciclofosfamida $50 \mathrm{mg} / \mathrm{kg} / \mathrm{día}$ y desde el día +5 , micofenolato y ciclosporina.

Resultados. Se trasplantaron 22 pacientes, edad media de 17 años (rango 4-31), el 14\% estaba en CR1; el 59\%, en CR2; y el 27\%, en CR3. Trece recibieron flu-bu-TBI y 9 flu-mel-TBI. Se infundió una media de 10 x 10(6) células CD34+/kg. La toma del injerto mieloide fue del $100 \%$, la recuperación de neutrófilos y plaquetas ocurrió en los días +15 y +17 . La incidencia de enfermedad injerto contra huésped $(\mathrm{EICH})$ aguda y crónica fue del $20 \%$ y $21 \%$, respectivamente. El seguimiento medio fue de 11 meses (rango 2-26), 5 pacientes murieron antes del día 100 por sepsis y 1 en el mes 26 por leucemia, otro más recayó al sexto mes y está en paliación. La sobrevida actuarial libre de leucemia (LFS) a dos años es del 55\%.

Conclusiones. La utilización de este protocolo de TMO-haplo-CFApos en pacientes con LLA mostró toma universal del injerto, aceptable incidencia de EICH, buena actividad antileucémica, solo dos recaídas y una halagadora LFS del $55 \%$. Es necesario mayor seguimiento y continuar la inclusión de pacientes.

\section{Evaluación de la densidad mineral ósea en pacientes con hemofilia A y B en Santander: estudio de casos y controles}

- Claudia Lucía Sossa Melo, Ángela María Peña Castellanos, Luis Antonio Salazar Montaña, Sara Inés Jiménez Sanguino, Juan Carlos Mantilla, Edwin Antonio Wandurraga Sánchez, Katherine Morales Chacón, Miguel Ángel Ayala Castillo, Miguel Enrique Ochoa Vera, Sandra Milena Acevedo Rueda

Fundación Oftalmológica de Santander, Carlos Ardila Lulle (Foscal), Floridablanca (Santander)

Contacto: claudiasossa@gmail.com

Introducción y objetivos. Establecer la asociación entre hemofilia y densidad mineral ósea (DMO) baja en hemofílicos A y B mayores de 5 años, comparado con controles en Santander. Evaluar los determinantes para baja masa ósea (BMO) en los casos: presencia de inhibidores, metabolismo fosfocálcico, marcadores de inflamación, perfil hormonal e infeccioso y actividad física.

Materiales y métodos. Estudio observacional tipo casos y controles. A todos se les realizó densitometría ósea (DXA) y medición del nivel de actividad física con el Global Physical Activity Questionnaire (GPAQ). Criterios de exclusión: hemofilia adquirida, enfermedad crónica, uso de esteroides, calcio, vitamina $\mathrm{D}$, antirretrovirales, tabaquismo y alcoholismo.

Resultados. Treintaiún casos y 31 controles. Edad promedio 26,8 años. La DMO de los casos fue inferior al grupo control en cadera ( $p$
$<0,01)$ y cuello femoral $(p<0,05)$. BMO para la edad en 10 casos $(32,2 \%)$ y 4 controles $(12,9 \%)$. Gradiente de asociación inversamente proporcional entre severidad de la hemofilia y la DMO. Asociación débil entre baja actividad física y DMO baja. Bajos niveles de $25(\mathrm{OH})$ D en el $29 \%$, alteraciones en calcio sérico del $9,6 \%$ y proteína $C$ reactiva elevada del $32,3 \%$. Veinte casos $(64,5 \%)$ con marcadores de resorción ósea elevados, 3 (9\%) menores de 18 años. Cuatro tenían inhibidores (12,9\%).

Conclusiones. Los hemofílicos tienen mayor riesgo de baja DMO en cadera y cuello femoral comparado con individuos sanos. Puede existir asociación entre severidad de la hemofilia y BMO. Los pacientes con hemofilia pueden presentar bajos niveles de vitamina D y marcadores de resorción ósea elevados. 